\title{
Adolescent sleep: a major public health issue
}

Fernando Louzada ${ }^{1}$

\author{
${ }^{1}$ Human Chronobiology Laboratory, \\ Department of Physiology, Federal \\ University of Paraná.
}

Twenty-six years ago, two seminal studies related to adolescent sleep were published. Andrade et al. ${ }^{1}$, in Brazil, and Carskadon et al. ${ }^{2}$, in the USA, obtained similar results: sleep phase delay in adolescents is associated with pubertal maturation. Since then, consequences and other factors associated with the delay have been depicted. In summary, sleep phase delay, combined with early school start times, results in chronic sleep restriction. This restriction is associated with several effects on daytime functioning, such as excessive daytime sleepiness, poor academic performance and increase in negative moods. Adolescent sleep has become a major public health issue. Countermeasures in order to attenuate undesirable consequences have been discussed and implemented. Basically, two main strands can be identified: sleep education and changes in school start times ${ }^{3}$. However, not much progress has been made in reducing sleep loss ${ }^{4}$. A significant percentage of teens has slept less than the preconized by the National Sleep Foundation recommendations ${ }^{5}$.

In this issue of Sleep Science, Lehto, Kortesoja and Partonen explore relationships between school burnout and daytime functioning. In their study, more than five hundred Finish students were evaluated through self-reports. School burnout was associated with daytime sleepiness, tiredness and poor sleep quality. Surprisingly, social jetlag was only weakly correlated to school burnout, and later bedtimes and wake-up times were not linked to burnout.

This issue also brings a study from Arbinaga, Joaquin-Mingorance and Fernández-Cuenca with more than one thousand Spanish university students showing an association between sleep quality and anger. Students with poor sleep quality had higher expression of anger. Consequences of sleep chronic sleep restriction are complex and reach cognitive and emotional domains.

Still in this issue, Mantua and collaborators discuss an effective countermeasure: in their study, sleep extension was able to reduce fatigue in healthy young adults. They suggest that this intervention may be beneficial to burnout.

Despite the recent efforts of the world's scientific community in order to improve adolescents' sleep, we still have too much work to do. Stakeholders must be better informed that we are facing a public health problem. Longitudinal studies must be conducted in order to test effects of countermeasures, such as changes in school start times. We have advocated that flexible school times could be an alternative, with the advantage of contemplating individual differences in diurnal preferences. Adolescents' developing brains and bodies need more sleep! We should not continue to deprive them of this.

\section{REFERENCES}

1. Andrade MM, Benedito-Silva AA, Domenice S, Arnhold IJ, Menna-Barreto L. Sleep characteristics of adolescents: a longitudinal study. J Adolesc Health. 1993;14(5):401-6.

2. Carskadon MA, Vieira C, Acebo C. Association between puberty and delayed phase preference. Sleep. 1993;16(3):258-62.

3. Louzada FM, Pereira SIR. Adolescents' sleep/wake patterns and school schedules: towards flexibility. Biol Rhythm Res. 2018;50(1):78-84.

4. Troxel WM, Wolfson AR. The intersection between sleep science and policy: introduction to the special issue on school start times. Sleep Health. 2017;3(6):419-22.

5. Hirshkowitz M, Whiton K, Albert SM, Alessi C, Bruni O, DonCarlos L, et al. National Sleep Foundation's sleep time duration recommendations: methodology and results summary. Sleep Health. 2015;1(1):40-3. 